\title{
Consideraciones sobre el marco regulatorio del peligro aviar en aeródromos de Honduras
}

Alex Matamoros

Carlos Roberto Torres

\section{Resumen}

La finalidad del presente artículo es hacer una revisión del marco normativo del peligro de impacto con fauna silvestre en aeropuertos y aeródromos, en tres niveles: en lo que corresponde la $\mathrm{OACl}$ como encargada de la aplicación y modificaciones al Anexo 14, en lo relacionado con las autoridades nacionales a quienes corresponde la emisión de Reglamentos de Aeronáutica Civil para los aeropuertos del País y por último en lo quecompete a cada uno de los aeropuertos quienes planifican, gestionan y ejecutan la aplicación práctica de la mencionada reglamentación. La Metodología utilizada se desarrolló en dos momentos, en primer lugar se profundizo en algunos de los aspectos abordados en el artículo "Identificación de los factores de atracción de fauna silvestre en las proximidades del Aeropuerto Toncontín"(Matamoros, 2015); en segundo lugar, se hizo una revisión del Anexo 14 y de la normativa aplicable a la prevención del peligro aviar en la aeronáutica. Se puede considerar como el principal resultado el haber hecho una primera aproximación a la condición en la que se encontraba el uso de la normativa consultada en el Estado de Honduras por parte de la Agencia Hondureña de Aeronáutica Civil y la Concesionaria Inter Airports administradora de los cuatro aeropuertos internacionales de Honduras, al momento de hacer el presente estudio. Se destaca de las conclusiones el llamado a desarrollar un trabajo conjunto entre la Autoridad Aeronáutica como ente regulador, la Concesionaria Inter Airport como encargada de los aeropuertos internacionales de Honduras y la UNAH, a través del Departamento de Biología quienes actualmente están finalizando un diagnóstico de la presencia de fauna en el Aeropuerto Ramón Villeda Morales de San Pedro Sula.

Palabras clave: Peligro por Fauna, marco regulatorio, planificación. 


\section{Abstract}

The purpose of this article is a revision of the regulatory framework of the impacthazard to wildlife in airports and aerodromes, on three levels: the first one corresponding ICAO Implementation and Amendments to Annex 14, the second level is in relation to the Nationals Authorities who are responsible for issuing Civil Aviation Regulations for the country's airports and, finally in relation to Each One of the Airports. The methodology used has been the Consultation in two phases, first it was made a review of some aspects of the related issues discussed at the report "Identification of the Factors Attracting wildlife near the Toncontin Airport" (Matamoros, 2015); also was made a review on the ICAO Annex 14 and on the applicable normative for the prevention of avian danger in the aviation. It can be considered the main result is have made a first approach to the status of implementation of the regulations consulted in the State of Honduras by the Honduran Civil Aviation Agencyand InterAiports, manager of Four International Airports in Honduras. An outstanding conclusions is the call to develop a joint work between the Aeronautical Authority As regulator, the Consecionaria Inter Airport as manager of the International Airports of Honduras and the National Autonomous University of Hondurasthrough the biology departmentwho arecurrently ending a diagnosis of the presence of wildlife in Ramón Villeda Morales airport in San Pedro Sula.

Keywords : Danger Wildlife, regulatory framework, planning

Alex Matamoros, (amatamoros@unah.edu.hn), Departamento de Ciencias Aeronáuticas, FACES/UNAH. Carlos Roberto Torres (Kriegsmarine21@gmail.com), Consultor Independiente en Temas Aeroportuarios. 


\section{INTRODUCCIÓN}

El documento de constitución de la OACl es el convenio de Chicago de 1944, después deeste año se han venido reglamentando los principales ámbitos de la aeronáutica mediante Anexos que contienen normas, definiciones y orientaciones operativas recomendadas; el Anexo 14 dedicado a la planificación, construcción y administración de aeródromos y helipuertos, en su volumen I Diseño y Operaciones de Aeródromos (OACl, 2009), reglamenta las medidas de mitigación del peligro por choque de aeronaves con fauna silvestre.

El propósito del presente artículo es hacer un segundo acercamiento al cumplimiento de la reglamentación $\mathrm{OACl}$ en Honduras en lo relacionado con peligro por fauna en los aeródromos, una primera aproximación fue hecha en una primera investigación, relacionada con la identificación de puntos de atracción de fauna en el Aeropuerto Toncontín de Tegucigalpa. Se cumple el propósito asignado, presentando los siguientes productos: 1)una síntesis de los requerimientos de cada uno de los principales documentos que contienen la reglamentación relacionada con el manejo de peligro por fauna; 2)un repaso de la forma en la que el Estado de Honduras ha adoptado la reglamentación presentada; 3 ) un resumen de los aportes de los participantes en la socialización de los resultados de investigación sobre puntos de atracción de fauna en Toncontin, hecha en la UNAH en 2015 4)un repaso de los requerimientos para la concesionaria Inter Airports y para la Autoridad Aeronáutica para atender el marco regulatorio sobre mitigación de riesgos de impacto de aves con aeronaves y 5) las conclusiones de la entrevista hecha al biólogo de la Concesionaria Inter Airports encargado de la mitigación del peligro por impacto de aves con aeronaves.

Partiendo del Anexo 14 como regla fundamental se estipulan para los países parte de $\mathrm{OACl}$, primero, un Manual de servicios de aeropuertos que en su parte 3 , regula la forma en que se debe mitigar el peligro que representan las aves para las operaciones de un aeródromo, en segundo lugar un manual para la sistematización de las notificaciones de impactos con aves; en base a estos documentos corresponde a las autoridades aeronáuticas nacionales establecer planes nacionales de mitigación del peligro de choque con aves en los aeropuertos nacionales; corresponde a los explotadores de aeropuertos y aeródromos elaborar programas de mitigación para cada uno de los aeropuertos administrados. La necesidad de previsiones por parte del Estado para la mitigación del peligro aviario y el llamado a la participación en estas iniciativas de distintos actores con presencia en el aeropuerto hacen el tema de fondo tratado en el presente artículo, una prioridad de estudio. 
Se cierra el artículo presentando cuatro conclusiones que evidencian la necesidad de continuar la presente investigación recogiendo información de los principales responsables de la aplicación de la reglamentación $\mathrm{OACl}$ en Honduras para conocer el nivel actual de aplicación del programa de mitigación del Aeropuerto Toncontín y las labores sobre prevención de peligro aviar de los demás aeropuertos. Esta revisión debe partir de dos supuestos: la Concesionaria se ha ocupado del tema de prevención y puede mejorar su desempeño, en cambio se constata una menor dedicación por parte de la Autoridad Aeronáutica que debe ser fortalecida institucionalmente para poder asumir su rol regulador.

\section{METODOLOGÍA}

\section{Revisión bibliográfica}

El punto de partida de la presente investigación fue una revisión bibliográfica de la reglamentación relacionada con el peligro aviar. El principal referente para este caso es el Anexo 14 de $\mathrm{OACl}$ sobre Aeródromos de $\mathrm{OACl}$ que expone un amplio abanico de temas relacionados con la planificación, construcción y aditamentos para el funcionamiento de aeropuertos y helipuertos. De esta base parten los documentos relacionados, revisados en este artículo, el Documento 9137 de OACl, denominado Manual de Servicios de Aeropuertos. Parte 3 Control y Reducción del peligro que representan la Fauna Silvestre; el Documento 9332 denominado Manual sobre el sistema de notificación de la $\mathrm{OACl}$ de los choques con aves, y la Regulación de Aeronáutica Civil RAC-139 de la Agencia Hondureña de Aeronáutica Civil, sobre Certificación y Operación de aeródromos.

\section{Revisión de los contenidos del primer trabajo de investigación}

Los contenidos de este artículo implicaron una revisión de una primera investigación que tuvo su énfasis en las condiciones presentes en el Aeropuerto Internacional Toncontín que pueden incentivar la presencia de fauna en la zona y que podría representar un peligro para las operaciones de las aeronaves en tierra o durante la aproximación, el aterrizaje o el despegue. En esa ocasión se contó con la colaboración de un especialista en temas aeroportuarios quien consideró que era oportuno hacer una nueva revisión del marco regulatorio y de una bibliografía representativa del campo, para establecer una base conceptual que fuera conocida y considerada en forma conjunta por AHAC y la UNAH; establecido un acuerdo sobre la reglamentación aplicable a este campo, se podrán examinar los niveles 
de cumplimiento alcanzados y el papel que correspondería a todos los actores involucrados en un proceso de seguimiento a la mitigación de condiciones que facilitan los impactos de aves en aeronaves a nivel nacional.

\section{Entrevistas a un especialista del tema y otras colaboraciones}

Se obtuvo la colaboración de dos especialistas, en primer lugar se aplicó una entrevista semi estructurada al Biólogo encargado de la prevención del peligro de impactos de aeronaves con fauna del Aeropuerto Villeda Morales de San Pedro Sula que tiene su sede de trabajo en este Aeropuerto y que cuenta con coordinaciones con el personal de seguridad aeroportuaria asignado a los demás aeropuertos que administra la concesión; en este caso se hizo una transcripción de la entrevista realizada para después aplicar a las respuestas una matriz de análisis para identificar las principales aportaciones y las conclusiones derivadas de las respuestas. El Segundo especialista es un consultor en temas aeroportuarios y sus colaboraciones se incorporan en el presente artículo como orientaciones para mejorar los aportes de los resultados y las conclusiones expuestas. El consultor mencionado se reporta como coautor del presente trabajo.

Fueron de mucha importancia los aportes derivados tanto de la experiencia del Biólogo de la Concesionaria quien lleva en esa labor más de tres años como del consultor quien ha laborado por más de cinco años en los aeropuertos internacionales de Honduras y ha presentado consultorías para distintos aeropuertos en Latinoamérica.

\section{RESULTADOS}

\section{Resumen de lo que proponen las reglamentaciones para el manejo del peligro aviar}

La prevención de incidentes y accidentes ocasionados por choques entre aves y aeronaves está contemplada en un marco regulatorio internacional el cual debe ser reflejado en una reglamentación interna de los estados partes de la $\mathrm{OACl}$. Posteriormente los operadores de aeropuertos deben asumir la planificación de medidas de mitigación de peligro por fauna por medio de programas de prevención para cada uno de los aeropuertos operados. Para la presente investigación se examinaron dos documentos que fungen como instructivos de reporte a nivel mundial y uno de aplicación nacional; los documentos internacionales son el Manual de Servicios de Aeropuertos (Doc 9137-AN898). Parte 3 Reducción del 
peligro que representan las avesy, el Manual sobre el sistema de notificación de la OACl de los choques con aves (DOC 9332), el documento nacional es la Regulación de Aeronáutica Civil. RAC-139. Certificación y operación de aeródromos. El documento 9137-AN898 toma en cuenta el imperativo del Anexo 14 de OACl sobre Aeródromos, según el cual los Estados están obligados a crear condiciones que minimicen la presencia de aves en los aeródromos. En este sentido ofrece orientaciones para crear un sistema que mitigue la presencia de aves en espacios de los aeródromos en donde puedan representar un peligro para las operaciones aeroportuarias (OACI 2012).

Tabla 1: Instancias de aplicación de Normativa OACl en el ámbito nacional (elaboración propia en base a Doc 9137 OACl).

\begin{tabular}{|c|c|c|}
\hline Instancia de coordinacion & Tipo de decisiones & Funciones \\
\hline Sede Nacional & Definición de políticas. & $\begin{array}{l}\text { Formula orientaciones, normas y directrices en consulta } \\
\text { con autoridades regionales. } \\
\text { Recomienda medidas biológicas y biotécnicas } \\
\text { aplicables a los entornos naturales de los aeropuertos. } \\
\text { Establece los comités de protección. }\end{array}$ \\
\hline Oficina regional & Dirección, técnica. & $\begin{array}{l}\text { Intermediar entre la Sede Nacional y el personal del } \\
\text { Aeropuerto. } \\
\text { Coordinación de actividades con las Autoridades } \\
\text { relacionadas con los aeropuertos. } \\
\text { Planifica actividades de mitigación de peligro a largo } \\
\text { plazo. } \\
\text { Ofrece recomendaciones técnicas para ejercer } \\
\text { actividades de control. }\end{array}$ \\
\hline Jefe de Aeropuerto & Técnica y operativas & $\begin{array}{l}\text { - Adoptar las medidas necesarias para la aplicación de } \\
\text { políticas de control del peligro aviar mediante } \\
\text { programa de limitación de avifauna. } \\
\text { - Hace seguimiento a las medidas programadas. } \\
\text { - Designa al coordinador de programa, un oficial de } \\
\text { control y un comité de control de peligro aviario. }\end{array}$ \\
\hline $\begin{array}{l}\text { Coordinador de programa y comité de } \\
\text { limitación de fauna silvestre. }\end{array}$ & Operativas & $\begin{array}{l}\text { - Reúne y capacita orienta a todas las dependencias } \\
\text { del aeropuerto que pueden colaborar en la restricción } \\
\text { de la presencia de aves. } \\
\text { Determina los responsables de cada una de las } \\
\text { oficinas implicadas en la restricción de presencia de } \\
\text { aves. } \\
\text { Coordina actividades de restricción de aves en el } \\
\text { aeropuerto. } \\
\text { Examina notificaciones de choque, informes de } \\
\text { mantenimiento y otros registros relacionados para } \\
\text { establecer planes de restricción de largo, mediano y } \\
\text { corto plazo. }\end{array}$ \\
\hline
\end{tabular}


La mitigación de la presencia de aves en el recinto aeroportuario es parte de un sistema de seguridad operacional cuyo buen funcionamiento está garantizado por la participación activa de los principales intervinientes de un sistema aeroportuario; en consecuencia es vital que todos reporten información fidedigna; esto incluye a pilotos, explotadores de aeronaves y en general, todo el personal que interactúa en el aeropuerto o aeródromo. Para efectos de brindar esta información OACI propone un formato de notificación de accidentes muy específico que incluye aspectos referentes a la aeronave impactada, las circunstancias en las que se dio el impacto, las partes de la aeronave afectada y las consecuencias para el vuelo, entre otras.

El Documento DOC 9332, por su parte, establece un sistema de notificaciones sobre choques entre una aeronave y una 0 varias aves con la intención de establecer un control para este tipo de colisiones y mantenerlo al alcance de los distintos usuarios interesados. Este sistema es explicado por medio de este manual en el que se describen entre otros conceptos y temas, las claves que se utilizan y los tipos de datos notificados. El modelo ofrecido, así como las codificaciones y los documentos a utilizar son sugeridos a los Estados, quienes pueden establecer, en base a lo propuesto, sus propios códigos y su propio sistema (OACl, 1990).

Por último la $R A C-139$, incorpora a la reglamentación de la aviación civil de Honduras lo establecido en el Volumen I del Anexo 14 de la OACI sobre certificación y operación de aeródromos; en tal sentido incluye una gama completa de aspectos que deben respetarse para la planificación, construcción, administración y manejo de aeródromos. También se trata el tema de reducción de peligro debido a las aves y otros animales, en el apartado RAC 139.337, en donde se establece que son obligaciones del operador de aeródromo: "Proveer o facilitar un estudio de fauna, a efecto de tomar las acciones pertinentes para minimizar los riesgos debidos a aves u otro tipo de animales" que identifique las especies existentes en el aeródromo, número, localidad, movimientos locales diarios y por estación, y ocurrencia de observaciones de animales; del aeródromo y de las cercanías del aeródromo la identificación, localización y demás aspectos descriptivos; la descripción de cualquier peligro de animales para las operaciones aéreas; el mecanismo de actualización periódica de información; los criterios de entidades públicas y privadas afectadas. Corresponde a la Agencia Hondureña de Aeronáutica Civil establecer si será necesario elaborar un plan de gestión de la fauna, éste plan debe contener, 1) personas encargadas del plan; 2) prioridades para el cambio de hábitat y los cambios del uso de la tierra identificada en el estudio de fauna, y fechas propuestas para cumplir el plan; 3) Información a ser enviada a las entidades públicas que 
emiten los permisos para el control de vida silvestre; 4) Identificación de los recursos que debe proveer el operador del aeródromo para la implementación del plan; 5) procedimientos y encargados de aplicarlos durante inspecciones físicas en áreas críticas por posibles peligros con animales, en dichas inspecciones se debe verificar medidas de control de animales, medios de comunicación efectiva entre el personal del operador del aeródromo, encargado del control de animales y los Servicios de Control de Tránsito Aéreo del aeródromo; 6) Procedimientos para la evaluación y revisión al menos cada 12 meses, los aspectos mínimos a evaluar serán, la efectividad del plan, las partes del plan que deben ser reevaluados, 7) un plan de entrenamiento para llevar a la práctica el plan. 8) los integrantes del comité de peligro aviar.

Se agrega que el operador del aeródromo debe mantener vigilancia para evitar la presencia de vertederos de basura con atractivo potencial para aves, en un radio de 13 kilómetros(8millas) en todas las direcciones del aeródromo; en el caso de que hubiese este tipo de vertederos se debe demostrar que no poseen atractivo, de lo contrario deberá notificar a la Autoridad Aeronáutica de la existencia de estos lugares por medio del Comité Nacional de Peligro Aviario y Fauna Silvestre. 
Tabla 2. Finalidad de los principales documentos para la aplicación de Normativa OACl.

\begin{tabular}{|c|c|c|}
\hline Documento & Finalidad & Entidad Responsable \\
\hline $\begin{array}{l}\text { 1. Manual de Servicios de } \\
\text { aeropuertos (Doc 9137) }\end{array}$ & $\begin{array}{l}\text { Orientar los Estados parte de } \mathrm{OACl} \text { en la creación de sistemas que } \\
\text { limiten la presencia de aves en espacios de los aeródromos en } \\
\text { donde puedan representar un peligro para las operaciones } \\
\text { aeroportuarias, recomendar la conformación de comités de } \\
\text { prevención y la planificación de mitigación de atractivos para aves. }\end{array}$ & $\begin{array}{l}\text { Organización de la Aviación } \\
\text { Civil Internacional. }\end{array}$ \\
\hline $\begin{array}{l}\text { 2. Manual sobre el } \\
\text { sistema de notificación } \\
\text { de la OACl de los } \\
\text { choques con aves } \\
\text { (DOC 9332) }\end{array}$ & $\begin{array}{l}\text { Establecer un sistema de notificaciones sobre choques entre una } \\
\text { aeronave y una o varias aves con la intención de establecer un } \\
\text { control para este tipo de colisiones; describen, las claves que se } \\
\text { utilizan y los tipos de datos notificados. El modelo ofrecido, asi } \\
\text { como las codificaciones y los documentos a utilizar son sugeridos a } \\
\text { los Estados. }\end{array}$ & $\begin{array}{l}\text { Agencia Hondureña de } \\
\text { aviación Civil y operadores de } \\
\text { aeropuertos. }\end{array}$ \\
\hline $\begin{array}{l}\text { 3. RAC-139. Certificación } \\
\text { y operación de } \\
\text { aeródromos. }\end{array}$ & $\begin{array}{l}\text { Incluye las obligaciones del operador de aeropuerto sobre } \\
\text { reducción de peligro debido a las aves y otros animales. }\end{array}$ & $\begin{array}{l}\text { Agencia Hondureña de } \\
\text { Aeronáutica Civil en } \\
\text { coordinación con operadores } \\
\text { aéreos. }\end{array}$ \\
\hline $\begin{array}{l}\text { 4. Plan Nacional de } \\
\text { prevención del peligro } \\
\text { de impactos con fauna } \\
\text { en aeropuertos. }\end{array}$ & $\begin{array}{l}\text { Definición u orientación de politicas que orienten las actividades y } \\
\text { tareas a desarrollar para la prevención de fauna en los aeropuertos; } \\
\text { implica ofrecer a los usuarios de la industria e institucionalidad } \\
\text { aeronáutica información que permita limitar la presencia de } \\
\text { avifauna en los aeropuertos; orientar la elaboración de planes de } \\
\text { manejo de fauna, diagnósticos del peligro que representan las aves } \\
\text { para las operaciones aéreas y aglutinar entes vinculados con la } \\
\text { prevención para acompañar procesos de ejecución de planes, entre } \\
\text { otras. }\end{array}$ & $\begin{array}{l}\text { Agencia Hondureña de } \\
\text { Aeronáutica Civil en } \\
\text { coordinación con operadores } \\
\text { aéreos. }\end{array}$ \\
\hline $\begin{array}{l}\text { 5. Programa de reducción } \\
\text { de impacto con fauna }\end{array}$ & $\begin{array}{l}\text { Presentar para cada aeropuerto administrado, las acciones y } \\
\text { procedimientos que corresponde a las instituciones y personas que } \\
\text { interactúan en este ámbito para prevenir y mitigar los riesgos } \\
\text { asociados a la presencia de fauna, atendiendo lo dispuesto por la } \\
\text { autoridad aeronáutica en el plan nacional; esto incluye una línea } \\
\text { base de la fauna existente en espacios en los que pueda } \\
\text { representar algún peligro para las operaciones aeronáuticas, los } \\
\text { componentes operativos del programa incluyendo, el } \\
\text { fortalecimiento de los actores involucrados para la gestión del } \\
\text { riesgo, la forma en que se llevaráa cabo el diagnóstico y monitoreo } \\
\text { del riesgo por fauna, el sistema de información para reportes, el } \\
\text { control y reducción del peligro ocasionado por la presencia de } \\
\text { fauna, la comunicación y capacitación y, los formatos para la } \\
\text { planeación y monitoreo del programa. }\end{array}$ & $\begin{array}{l}\text { Operador aéreo encargado del } \\
\text { aeropuerto } 0 \text { aeródromo. }\end{array}$ \\
\hline
\end{tabular}

Fuente: Elaboración propia en base a documentos normativos y operativos.

\section{Adopción de la reglamentación por las Autoridades Nacionales}

Una vez adoptada la reglamentación internacional sobre peligro aviar, corresponderá a las Autoridades Aeronáuticas la conducción de las medidas necesarias para su implementación en términos operativos. La función principal de la Autoridad Aeronáutica con respecto a la prevención de impactos por aves en aeropuertos es 
el establecimiento de un plan nacional que operativice todo lo relacionado con el tema. Entre los objetivos de un plan de prevención está en primer lugar la definición u orientación de políticas que orienten las actividades y tareas a desarrollar para la prevención de la presencia de fauna en los aeropuertos; a esta finalidad básica se pueden agregar los objetivos de ofrecer para usuarios específicos de la industria e institucionalidad aeronáutica información que permita la presencia de avifauna en los aeropuertos, orientar la elaboración de planes de manejo de fauna, incentivar y orientar diagnósticos del peligro que representan las aves para las operaciones aéreas, orientar la elaboración de inventarios de los factores de atracción de avifauna en los aeropuertos, divulgar la normativa relacionada con el tema y aglutinar a las entidades y personas vinculadas con la prevención para acompañar procesos de ejecución de planes, entre otras. En síntesis este plan es "una guía completa de las políticas adoptadas por la Entidad (la Autoridad aeronáutica) para la prevención de los impactos entre aeronaves y representantes de la fauna" (Unidad Administrativa especial de Aeronáutica Civil, Colombia, 2008).

Una de las modalidades que pueden asumirse para la incorporación de la reglamentación internacional sobre peligro por fauna, es la edición de guías para la elaboración de estudios de riesgo de impactos con aves en Aeropuertos. Este tipo de guías ofrece orientaciones para la elaboración, por parte de los operadores aeroportuarios, el análisis de riesgo de impactos de aves con aeronaves. Se aporta una metodología para identificar los riesgos existentes y aplicar las medidas de mitigación de los riesgos identificados. Se dice que los resultados del estudio generado deben integrarse a los sistemas de gestión de riesgo del aeropuerto y ser revisados periódicamente para actualizar sus datos y medir la eficacia de las medidas derivadas de las conclusiones y recomendaciones ofrecidas (AENA, 2013). Según la guía el informe debe contemplar como contenido sustancial, un diagnóstico y análisis de ámbito de estudio, la identificación de los peligros encontrados en los términos que lo establece la reglamentación correspondiente, una descripción analítica de los riesgos percibidos y documentados en la que se incluyan hallazgos que cuantifiquen las probabilidades de colisión y la gravedad de los daños reales 0 potenciales, el análisis también incluye el establecimiento de niveles de riesgo; se espera además que el informe contenga el detalle de las medidas de mitigación de los riesgos reportados y analizados, dichas medidas deben ocuparse al menos de la gestión del hábitat, la exclusión, expulsión y captura o eliminación de fauna, así como de otras medidas consideradas necesarias. Dos aspectos más a incluir en el informe son la periodicidad con la que se evaluará la aplicación de medidas en términos de actualización de datos y efectividad de las medidas implementadas y las conclusiones. 
Además se puede reglamentar el desarrollo de estudios específicos aplicados al peligro aviar, en cuyo caso al protocolo de investigación se agrega la inclusión de datos estadísticos de la presencia de aves, incluyendo números aproximados de sujetos, lugares de avistamiento, fechas y horas del día de su avistamiento, características de las especies observadas, entre otros (AENA, 2013). Este tipo de estudios requieren también el análisis de los riesgos generados por presencia de aves y una propuesta de medidas de mitigación del peligro en términos de gestión del hábitat del entorno aeroportuario, exclusión y expulsión de fauna, captura 0 eliminación; así como periodicidad.

Sin embargo, todas las actividades de prevención que se lleven a la práctica en cada uno de los aeródromos que se rijan por un plan nacional de prevención, se deben reflejar en un programa de reducción de impacto con fauna que tome en cuenta las características específicas del aeropuerto para el cual fue diseñado. Se trata de un documento técnico específico que presenta las acciones y procedimientos que corresponde a las instituciones y personas que interactúan en un aeropuerto para prevenir y mitigar los riesgos asociados a la presencia de fauna en un aeródromo 0 aeropuerto, atendiendo lo dispuesto por la autoridad aeronáutica en el plan nacional. Normalmente este documento incluye una línea base de la fauna existente en espacios en los que pueda representar algún peligro para las operaciones aeronáuticas, es un "diagnóstico de amenaza por presencia de fauna silvestre peligrosa para la aviación" (Restrepo-Calle, 2009, citado en OPAIN, 2010); seguidamente se presentan los componentes operativos del programa incluyendo, el fortalecimiento de los actores involucrados para la gestión del riesgo, la forma en que se llevará a cabo el diagnóstico y monitoreo del riesgo por fauna, el sistema de información para reportes, el control y reducción del peligro ocasionado por la presencia de fauna, la comunicación y capacitación; por último se establecen los formatos para la planeación y monitoreo del programa.

\section{Aportes de los participantes en la socialización de los resultados de investigación sobre puntos de atracción de fauna en Toncontín}

Durante el año 2015 en ocasión de la socialización en la UNAH de la investigación "Identificación de los factores de atracción de fauna silvestre en las proximidades del Aeropuerto Internacional Toncontín", se tuvo la oportunidad de intercambiar opiniones con distintos profesionales y técnicos relacionados con el tratamiento desde la práctica del peligro aviar en aeropuertos. Entre otros participantes se contó con la presencia de personal encargado de la seguridad operacional de Toncontín y con un docente que a la fecha se encontraba apoyando por parte del 
Departamento de Biología de la UNAH, el levantamiento de un inventario de la fauna en el Aeropuerto Internacional Ramón Villeda Morales de San Pedro Sula, hasta aquel momento la investigación se encontraba más o menos a la mitad de su elaboración. A continuación se presentan los principales aportes hechos por los participantes en este evento:

- Una representante de la Concesionaria Inter Airports explica que este consorcio ya cuenta con un programa de mitigación del peligro aviar para el Aeropuerto Toncontín y que se tiene noticia del proceso de elaboración que se está llevando a cabo en los otros tres aeropuertos internacionales, se recuerda que los cuatro Aeropuertos están siendo administrados por una misma Concesionaria. Al momento de la participación, el programa de Toncontín, se encontraba en la fase de implementación, por lo cual el expositor explicaba que era este el mejor momento para incorporar a todos los actores relacionados con la prevención de incidentes y accidentes por colisión de aves con aeronaves, puesto que la mayor participación es un garantía de éxito en términos de eficiencia en la aplicación del programa.

- De otros comentarios se constata la centralidad de la colaboración del Estado, puesto que este debe asumir su rol de generador y gestor de políticas por medio de un Sistema Nacional de prevención al cual deben orientarse todos los programas de prevención, de los cuales debe existir uno por cada aeropuerto.

- Ilustrando de otra manera la centralidad del estado en el proceso de adopción de la reglamentación internacional sobre prevención del peligro aviar se explica que en el caso de Toncontín uno de los principales puntos de atracción de aves y otros animales es la base de la Fuerza Aérea, debido a la poca atención que se presta al manejo adecuado de sus áreas verdes; por otra parte la Concesionaria no puede por sí sola provocar la remoción de los depósitos de residuos que podrían representar otro atractivo importante para aves, roedores y otros animales; corresponde al Estado la reubicación o la vigilancia de los depósitos de basura y el ordenar a la Fuerza Aérea para que mejore el mantenimiento de las propias áreas verdes.

- Se comenta que una forma de implementar los programas de prevención en cada uno de los cuatro aeropuertos internacionales de Honduras, garantizando en alguna medida la correcta implementación en términos de efectividad, es capacitar a los actores directamente involucrados con el apoyo de especialistas con experiencia; lo ideal sería que se replicara la formación recibida por los encargados de seguridad operacional de los cuatro aeropuertos internacionales, 
incluyendo visitas de campo atendidas por equipos interdisciplinarios con integrantes de las empresas y de la institucionalidad del Estado relacionada directa o indirectamente con el tema de seguridad operacional aeronáutica.

\section{Condiciones necesarias para atender los requerimientos de un marco regulatorio sobre mitigación de riesgos de impacto de aves con aeronaves}

En el caso de Honduras se puede hacer una lista de instituciones y profesionales relacionados con el tema de prevención de peligro aviar que incluiría pilotos, líneas aéreas, servicios de groundhandling, las escuelas de vuelo, entre otras; sin embargo, consideramos que dado el momento que se está viviendo de inicio de implementación del programa de prevención, el grueso de las responsabilidades recae sobre dos grandes actores: la Agencia Hondureña de Aeronáutica Civil y la Concesionaria Inter Airports, ambos entes tendrían que conformar un núcleo que incorporé activamente a los demás actores.

Por su parte la Concesionaria está cumpliendo con lo que le corresponde incorporando la prevención del peligro aviar dentro de su sistema de manejo de la seguridad en las operaciones (SMS por sus siglas en Inglés), asignando un biólogo encargado para este tema que aunque tiene sede en San Pedro Sula está coordinando a nivel nacional, y dando la formación oportuna y necesaria a su personal para atender el tema, de hecho se tiene noticia de que el personal de seguridad ha sido capacitado por el profesional que conduce el proceso de programa de prevención en el Aeropuerto Internacional de Comalapa en El Salvador, se sabe que el entrenamiento incluyó visitas a los lugares del entorno aeroportuario en donde existe mayor potencial de choques entre aves y aeronaves en el entorno del aeropuerto Toncontín.

A nivel de País le corresponde a la Agencia Hondureña de Aeronáutica Civil tomar la iniciativa y ejercer control sobre el tema, sin embargo se constatan una serie de limitantes que obstaculizan a esta institución asumir su rol:

- El país no cuenta con un Sistema Nacional de Gestión de la Seguridad Operacional dentro del cual se debería incluir un capítulo dedicado al peligro aviar, para cumplir así lo establecido en el RAC 139 con respecto al tema, en términos de exigir cumplimiento a la Concesionaria.

- Se debe contar con un programa de auditorías de cumplimiento de los programas de prevención que deberían estar operando en los cuatro 
aeropuertos internacionales de Honduras; hasta ahora las evaluaciones de estos programas deben ser hechas por la misma concesionaria.

- Por lo visto no se cuenta con personal entrenado para que divulgue la importancia y la necesidad de contar con medidas programadas de prevención de choques entre aves y aeronaves; esto implicaría no sólo el conocimiento de los formatos de reporte que deben ser llenados en los distintos niveles, sino también la aplicación de auditorías de cumplimiento de medidas.

- Es una obligación del Estado, como lo indica el Documento 9137 de $\mathrm{OACl}$, entregar reportes periódicos de impactos y se sabe que estos reportes han sido entregados como está establecido, sin embargo, los datos recabados no están siendo utilizados como insumo para analizar los alcances que pueden tener los impactos, hasta ahora no se percibe periodicidad en la entrega de esta información a los usuarios nacionales y la Autoridad Aeronáutica sólo ha hecho comparecencias cuando se han suscitado incidentes considerables. En opinión de los autores del presente artículo, un aspecto que se debe cuidar en este caso es el de no divulgar datos o información que pueda ser manipulada por los medios de comunicación afectando negativamente el manejo de la seguridad en los aeropuertos al atribuir al concesionario, la responsabilidad por los incidentes 0 accidentes.

\section{Principales aportes de la entrevista realizada al encargado de fauna de Inter Airports.}

La concesionaria Inter Airports se ha ocupado del tema de prevención del peligro por fauna en aeropuertos desde hace al menos tres años, es decir desde el momento en que contrató por primera vez un biólogo para colocarlo como encargado del tema. Este profesional fue entrevistadoen el Año 2014 ya continuación se ofrece un resumen los principales aportes hechos de esta consulta:

1. El trabajo en liderar, dirigir y gestionar está recayendo principalmente sobre el biólogo que sólo en el caso del Aeropuerto de San Pedro Sula cuenta con un equipo de apoyo.

2. A partir de los contactos que se han hecho hasta la fecha, se puede establecer un equipo de trabajo que contaría con la participación de un amplio espectro de instituciones relacionadas con el tema. 
3. El contacto que se ha hecho con varios actores involucrados o por involucrarse en el tema facilitará la elaboración de planes estratégicos y operativos en este campo.

4. Se entiende que el peligro aviar es mayor en Tegucigalpa por la existencia de numerosos botaderos situados en zonas subyacentes al cono de aproximación al aeropuerto.

5. Solamente con zopilotes se han dado incidentes de los que se tiene registros, pero no una estadística específica.Para conocer datos sobre la potencialidad de peligro de otros tipos de aves se tendría que hacer otro tipo de estudios, para los cuales por ahora no se tiene una disposición de recursos.

6. La obligación de respetar la biodiversidad, no parece ser un obstáculo significativo en la labor de disminuir el peligro aviario puesto que cuando se presentó, se pudo solventar con facilidad.

\section{DISCUSIÓN}

La aeronáutica en lo que respecta a los aeropuertos es un campo con reglamentación suficiente para contar con orientaciones y eventualmente indicaciones para las actividades operativas más importantes; esta aseveración se cumple también con la prevención del peligro aviar que cuenta con una base conceptual y orientaciones para la elaboración de políticas, muy claras en el anexo 14 de la OACl. Esta claridad se refleja además en los documentos que se derivan del mencionado anexo, guían su aplicación desde los más altos niveles, es decir la $\mathrm{OACl}$ como instancia de la Naciones Unidas, hasta los niveles puramente operativos, es decir el personal encargado de seguridad de los aeropuertos.

Los elementos a tomar en cuenta para establecer un programa de prevención del peligro que puede representar la fauna para un aeropuerto son múltiples; sin embargo, dados los costos que pueden acarrear las actividades implicadas es de esperarse que sean determinantes las variables relacionadas con la rentabilidad del aeropuerto o aeródromo en cuestión. Esto constituye un primer obstáculo de implementación de las medidas a proponer puesto que existen aeródromos en el país que funcionan en contextos geográficos ricos en flora y fauna que no cuentan con una rentabilidad que permita el establecimiento de los controles necesarios para mitigar el peligro por aves. 


\section{CONCLUSIONES}

1. La presente investigación fue una revisión prevalentemente bibliográfica a partir de la cual se pueden elaborar instrumentos de consulta para examinar el tema de la prevención de peligro de choque entre aeronaves y fauna en los aeropuertos del País, consultando una muestra significativa de los intervinientes que interactúan en nuestros aeropuertos. En una primera acotación de entrevistados se considera relevante la información que pueden ofrecer: 1)la Concesionaria de aeropuertos en lo que respecta a la planificación operativa de prevención, 2) la Agencia Hondureña de Aeronáutica Civil en lo relacionado con la emisión de políticas de aplicación nacional y supervisión de su aplicación; 3) El departamento de Biología de la UNAH por lo que tiene qué ver con la información diagnóstica que están levantando para el Aeropuerto Internacional Ramón Villeda Morales de San Pedro Sula.

2. Se tiene conocimiento de la existencia del comité de peligro aviar del Aeropuerto Internacional Toncontín desde el Año 2014, esto hace suponer que existe un programa que sin duda fue elaborado según las directrices de la reglamentación internacional; en este sentido, es ahora un buen momento para que la Agencia Hondureña de Aeronáutica Civil, realice una evaluación de la aplicación de este plan, de la cual se obtendría valiosa información para establecer un estado del arte de la aplicación de la reglamentación internacional sobre peligro por fauna en lo concerniente tanto al Estado como al único concesionario de aeropuertos que existe en la actualidad.

3. Analizar la aplicación de la reglamentación OACI sobre peligro por fauna, requiere la recolección de información de un período de al menos cinco años, lo cual implica examinar para este período, en el personal encargado de seguridad y en la infraestructura de servicios aeroportuarios y de información, aspectos como el conocimiento de la reglamentación, el fortalecimiento institucional de los entes encargados de la prevención del peligro por fauna en aeropuertos, la capacitación y el entrenamiento del personal encargado directamente, la elaboración y aplicación de un plan nacional de prevención de peligro aviar o bien de las directrices emitidas por la Autoridad Aeronáutica en el período mencionado y el seguimiento de las actividades de prevención dentro de los programas de cada aeropuerto, entre otros. dada la diversidad de aspectos a revisar este ejercicio de recolección y análisis de información necesita como condición imprescindible, la amplia colaboración de la Autoridad Aeronáutica y del actual concesionario. 
4. La correcta aplicación de la reglamentación $\mathrm{OACl}$ sobre mitigación de peligro por presencia de fauna en el ámbito de las operaciones aeronáuticas en los aeropuertos internacionales de Honduras, significaría para la única concesionaria un esfuerzo por mejorar su ya existente sistema de gestión de la seguridad operacional en lo que respecta a la prevención de impactos de aeronaves con fauna, lo cual es factible por cuanto la concesionaria cuenta con un buen sistema; en lo que respecta a la Agencia Hondureña de Aeronáutica Civil, se notan falencias más difíciles de sobrellevar dada la poca atención que se ha prestado al tema.

\section{AGRADECIMIENTOS}

Este trabajo no habría sido posible sin la colaboración del consultor independiente nombrado como coautor de este trabajo, este profesional hizo importantes aportes para mejorar los resultados y puntualizar mejor las conclusiones; igual de importantes fueron los aportes obtenidos en la entrevista al biólogo asignado al Aeropuerto Internacional Ramón Villeda Morales de San Pedro Sula y encargado de la planificación de la mitigación del peligro de choques con fauna en los cuatro aeropuertos internacionales de Honduras. Se agradece a ambos su generosa colaboración. De igual manera se agradece a la Concesionaria Inter Airport por facilitar el tiempo de uno de uno de sus empleados técnicos y a la Facultad de Biología de la UNAH, por compartir con el Departamento de Ciencias Aeronáuticas, la presentación de los resultados parciales del diagnóstico sobre presencia animal en el Aeropuerto Ramón Villeda Morales de San Pedro Sula.

\section{BIBLIOGRAFÍA}

- AENA . (2013). Guía para la elaboración de estudios de riesgos de impacto con aves en aeropuertos. Madrid: AENA.

- DGAC. (2008). Regulación de Aeronáutica Civil. RAC-139. Certificación y operación de aeródromos. Tegucigalpa: DGAC.

- Matamoros, A. (2015). Identificación de los factores de atracción de fauna silvestre en las proximidades del Aeropuerto Toncontín. Revista Ciencias Espaciales, Otoño 2014. Tegucigalpa.

- OACl . (2012). Manual de Servicios de Aeropuertos (Doc 9137). Parte 3 Control y Reducción del peligro que representa la fauna silvestre. Montreal: $\mathrm{OACl}$. 
- OACl. (199). Manual sobre el sistema de notificación de la OACl de los choques con aves (DOC 9332). Montreal: OACl.

- OACI. (2009). Anexo 14, Volumen I Diseño y operaciones de aeródromos. Montreal : $\mathrm{OACl}$.

- OPAIN. (2010). Programa de reducción de impactos con fauna Aeropuerto Internacional El Dorado. Bogotá: OPAIN.

- Restrepo-Calle, S. (2009). Implementación de un programa de control integral de fauna silvestre en el Aeropuerto Internacional de El Dorado. Fase I -Actualización diagnóstico de amenaza por presencia de fauna silvestre peligrosa para la aviación. . Bogotá: OPAIN.

- Unidad Administrativa especial de Aeronáutica Civil, Colombia. (2008). Programa Nacional de limitación de fauna en Aeropuertos. Bogotá: Unidad Administrativa Especial de Aeronáutica Civil. 